\title{
Optimal Synthesis of Feeding Network for Implementation of Dolph-Chebyshev Current Distribution on Microstrip Antenna Arrays
}

\author{
Ioannis P. Gravas, Student Member, IEEE, Zaharias D. Zaharis, Senior Member, IEEE, Traianos V. \\ Yioultsis, Member, IEEE, Pavlos I. Lazaridis, Senior Member, IEEE, Keyur K. Mistry, Student Member, \\ IEEE, and Thomas D. Xenos
}

\begin{abstract}
A microstrip feeding network (MFN) that implements a Dolph-Chebyshev current distribution is designed to feed a microstrip antenna array (MAA) operating in B2 LTE band. The study consists of three phases. In the first one, the elements of MAA are directly excited by equi-phase current sources complying with a Dolph-Chebyshev amplitude distribution to ensure a side lobe level (SLL) of $-20 \mathrm{~dB}$. Then, MAA is optimized for maximum forward gain. Finally, the input impedances of the elements of the optimized MAA and the element spacing are recorded. In the second phase, the MFN is considered to terminate at lumped loads with values equal to the input impedances of the respective elements of the optimized MAA, and is then optimized to achieve low standing wave ratio, high power efficiency, and output currents equal to those applied in the first phase by the current sources. All optimizations are performed with an improved particle swarm optimization variant in conjunction with CST. In the third phase, the optimized MFN is attached to MAA and is evaluated with CST. The purpose of this study is to show that it is possible to design an MFN that satisfies multiple requirements, without the knowledge of MAA geometry.
\end{abstract}

Index Terms-Antenna arrays, Dolph-Chebyshev distribution, microstrip lines, particle swarm optimization.

\section{INTRODUCTION}

Microstrip feeding networks (MFNs) provide an efficient way to achieve specific types of excitations (amplitudes and phases) on microstrip antenna arrays (MAAs) and have a wide range of applications in printed antennas and array feeding [1]-[14]. A Dolph-Chebyshev (D-Ch) excitation distribution enables the antenna array to produce a radiation pattern with the desired side lobe level $(S L L)$ [15]. Usually, an $S L L$ of $-20 \mathrm{~dB}$ is considered to be sufficient to restrict the amount of power wasted due to spatial spread of radiation. A common practice to design feeding networks that implement excitation distributions of non-uniform amplitudes is the use of resistive components. However, this practice is not energy-efficient, because resistive components absorb power and consequently their use results in significant power loss. An efficient solution is the use of a specific microstrip geometry defined by a certain number of microstrip transmission line segments, which have proper lengths and widths, and are connected to each other according to a particular topology, without using any resistive components at all. The definition of this geometry is a very complex problem in practice due to the mutual coupling among the array elements, and also between
MAA and the MFN. To include the mutual coupling effect in the electromagnetic (EM) behavior of the MFN, full wave analysis must be applied. For this purpose, we employ the time domain solver of the CST Microwave Studio (CST MWS) software package [16]. Also, proper lengths and widths of the transmission line segments that compose the MFN can be found by applying evolutionary optimization algorithms. To the best of the authors knowledge, evolutionary algorithms have never been applied so far to design power-efficient MFNs that implement a D-Ch excitation distribution at their output ports under realistic conditions.

In this study, an MFN is designed and optimized in order to create output currents that follow a D-Ch amplitude distribution by exploiting the properties of standing waves inside the microstrip lines and without using resistive components. Due to the absence of resistive components, the proposed MFN is expected to exhibit high power efficiency. The MFN is optimized separately from MAA to show that a proper MFN, which creates output currents following a D-Ch amplitude distribution, can be designed for any MAA, provided that we know the input impedance of each array element in the presence of the other elements (not the self-impedance) and the element spacing.

The whole study consists of three phases, which are described in detail below. The first phase does not concern the design of the MFN but aims at finding the geometry of a MAA that produces a radiation pattern with $S L L=-20 \mathrm{~dB}$ and maximum forward gain $(F G)$. Such a MAA could be a commercial antenna array utilized by wireless communications base stations. Since the geometry and radiation characteristics of a base station antenna array were not available in this study, we decided to model such an array in CST MWS. To ensure an $S L L$ of $-20 \mathrm{~dB}$, the array elements are considered to be directly excited at their input ports by sources, which create input currents following a D-Ch amplitude distribution and have equal phases (thus producing a broadside radiation pattern). Then, MAA is optimized only for maximum $F G$. In the end of this phase, the input impedance of each element (in the presence of the other elements ) of the optimum MAA and the element spacing are recorded to be used in the optimization of the MFN.

In the second phase (i.e., the main phase), the MFN is considered to terminate not at the elements of MAA but at lumped loads with values equal to the input impedances 
of the respective elements of the optimum MAA. With this consideration, the proposed MFN design procedure provides the great advantage that the exact geometry of MAA is not required to find the optimum MFN geometry. Subsequently, the MFN is optimized to achieve:

1) standing wave ratio $(S W R) \leq 1.2$,

2) power efficiency $(P E) \geq 80 \%$,

3) amplitudes of output currents (on the lumped loads) following a D-Ch distribution that corresponds to $S L L=$ $-20 \mathrm{~dB}$, and

4) phase difference between any two output currents $\leq 3^{\circ}$. It must be mentioned that the 3rd requirement is quite strict because it demands specific values for the amplitudes of the output currents. To help the optimization algorithm achieve these values, we decided to define a loose (4th) requirement for small phase differences between the output currents instead of demanding equiphase currents. A proper description of the 1 st, 2 nd and 4 th requirement in the definition of the respective fitness function also helps the optimization algorithm to satisfy the 3rd requirement, as explained in Section V.

In the third phase (i.e., the evaluation phase), MAA is attached to the optimum MFN, the whole structure is analyzed with CST MWS, and the results are evaluated in comparison to those extracted in the previous phases.

To optimize MAA (phase 1) or the MFN (phase 2), we employ a particle swarm optimization (PSO) variant, called PSOvm [17], [18] which induces mutation to the particles' velocities that did not improve their fitness in the previous iteration. The idea behind this method is to decouple from its previous velocity every particle that did not manage to improve its fitness (inactive particle). Thus, a perturbation is applied to the velocity that the inactive particle had in the previous iteration, which actually did not lead to a better fitness. PSOvm has been proven to be competitive against other well-known evolutionary methods [17], [18] and can effectively handle multiple-variable non-linear optimization problems, as is this case.

For each fitness evaluation required by PSOvm, a full wave analysis is performed (on MAA in phase 1 or on the MFN in phase 2) by applying CST MWS. Due to the complexity of the MFN geometry, each fitness evaluation during the MFN optimization is costly in terms of resources and time, but the accuracy of the results is high. The computer used for the simulations was equipped with an Intel i7 5960X (eightcore) CPU with 64 GB DDR4 memory. The CST models created for the optimization procedures of phases 1 and 2 consist of around 200,000 mesh cells (as the model changes its dimensions at each fitness evaluation, the amount of mesh cells changes as well), while the average computation time was around half a minute and two minutes for phase 1 and phase 2, respectively. Phase 1 concluded after 200 fitness evaluations, while phase 2 after 1200 evaluations.

\section{PRIOR ART}

Prior to our study, an embedded corporate MFN has been designed in [19]. The array elements and the MFN were in different substrate layers and they were powered by parasitic coupling through the substrate. The phases and the amplitudes of the current distribution were predicted by using network theory and by taking into account the discontinuities, while $P E$ of the MFN was not considered in the MFN design. A dual-band (900 MHz and $1800 \mathrm{MHz}$ ) base station wire antenna array has been presented in [20] together with two types of feeding networks that implement in both bands a Chebyshev current distribution at the feed points of the antenna array. The first feeding network makes use of short-circuited stubs resulting in narrow bandwidth resonances in $900 \mathrm{MHz}$ and $1800 \mathrm{MHz}$. The second feeding network solves the narrow resonances problem by using stubs terminated in lumped loads but the power efficiency of the network is degraded. An MFN for aperture coupled MAA has been designed in [21] using T-junctions and Wilkinson power dividers to achieve D-Ch power ratios. T-junction power dividers have also been used in [22] to design an MFN that achieves a D-Ch current distribution on its output ports, but without mentioning anything about the current phases. An MFN has been designed in [23] to produce a D-Ch excitation distribution for a $24 \mathrm{GHz}$ MAA. A D-Ch current distribution was used in [24] to excite an $8 \times 8 \mathrm{MAA}$. To separate MAA from the MFN, multi-layer substrates were used, while the excitation was applied by using electromagnetically coupled patches and L-probe feeding, but without mentioning anything about the current phase differences. A four-element ultra-wideband MAA has been designed in [25]. The feeding network was composed of Wilkinson power dividers in order to achieve DCh amplitude distribution. A dual-polarized planar MAA with in series-parallel feed configuration has been designed in [26] in order to implement D-Ch amplitudes distribution to reduce the SLL. An 144-element MAA has been designed in [27] and implements D-Ch amplitude distribution by using T-junction power dividers. Dual substrate has been used to separate the MFN from MAA and the elements were connected to the MFN through vias.

Feeding network designs, like those described in [19], [21], [24] and [27] help to reduce the coupling between the array elements and the feeding network, but have difficulty in fabrication and are more expensive. Also, in all the above mentioned studies, evolutionary optimization algorithms were not used to adjust the geometry of the feeding network. Contrary to the above MFNs, the proposed MFN structure can easily be fabricated, while it achieves equiphase currents with amplitudes following a D-Ch distribution (thus it ensures $S L L=-20 \mathrm{~dB}$ ), and concurrently obtains optimal values of $P E$ and $S W R$. Finally and most importantly, our proposed method works for every MAA, given that the input impedances (not the self-impedances) of the array elements and the element spacing are known as previously explained.

\section{GeOMETRY DEFINITION}

\section{A. Microstrip Antenna Array Geometry}

Data concerning the geometry and EM behavior of a base station MAA were not available in this study, as mentioned in section I. Therefore, CST MWS was employed to model a linear antenna array composed of eight rectangular microstrip 


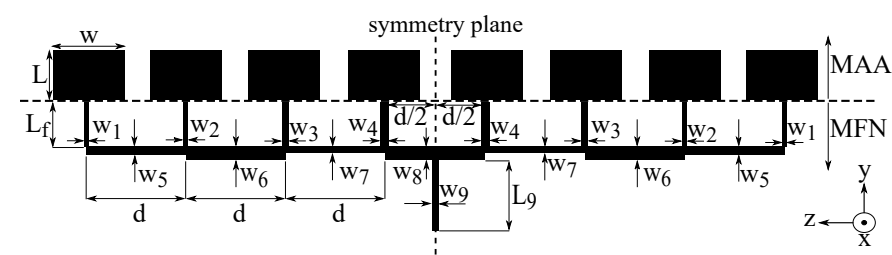

Fig. 1. Geometry of the proposed Dolph-Chebyshev feeding network (the subscript of a segment width denotes the sequence number of the segment).

elements, which are directly excited at their input ports by sources, i.e., without using a feeding network. The array is designed for operation in B2 LTE band $(1900 \mathrm{MHz})$. To achieve a broadside radiation pattern with $S L L=-20 \mathrm{~dB}$, the currents at the input ports of the elements must have equal phases and amplitudes following a D-Ch distribution.

MAA is illustrated in Fig. 1 and its geometry is defined by the physical dimensions $w$ (width) and $L$ (length) of every element, and element spacing $d$. As indicated in [28], the theoretical dimensions $w_{t h}$ and $L_{t h}$ of rectangular microstrip elements are respectively given by:

$$
w_{t h}=\frac{c}{2 f_{r}} \sqrt{\frac{2}{\epsilon_{r}+1}}
$$

and

$$
L_{t h}=\frac{c}{2 f_{r} \sqrt{\epsilon_{r e f f}}}-2 \Delta L,
$$

where $c$ is the free space wave velocity, $f_{r}$ is the operation frequency (1900 MHz in this study), $\epsilon_{r}$ is the dielectric constant of the substrate used here, $\epsilon_{r e f f}$ is the effective dielectric constant, and $\Delta L$ is the length reduction applied at both sides of the effective length to get physical length $L$. The values of $\epsilon_{r e f f}$ and $\Delta L$ are respectively estimated [28] by:

$$
\epsilon_{\text {reff }}=\frac{\epsilon_{r}+1}{2}+\frac{\epsilon_{r}-1}{2}\left[1+12 \frac{h}{w_{t h}}\right]^{-1 / 2}, \frac{w_{t h}}{h}>1
$$

and

$$
\Delta L=0.412 h \frac{\left(\epsilon_{\text {reff }}+0.3\right)\left(w_{t h} / h+0.264\right)}{\left(\epsilon_{\text {reff }}-0.258\right)\left(w_{t h} / h+0.8\right)},
$$

where $h$ is the substrate height (or thickness). The substrate used in this study is Duroid RT5880 with $\epsilon_{r}=2.2$ and $h=$ $3.175 \mathrm{~mm}$ [29]. The thickness of the metallic strips above the substrate is equal to $35 \mu \mathrm{m}$ [29] (to be used in CST modeling). By applying the above equations, we get $w_{t h}=62.4 \mathrm{~mm}$ and $L_{t h}=51.5 \mathrm{~mm}$.

PSOvm [17] is applied in the first phase to find optimal values for $w, L$ and $d$ ( $Q=3$ optimization parameters) that maximize $F G$. To help PSOvm converge faster, $w, L$ and $d$ must be restricted between a lower and an upper limit. By considering that the optimal values of $w$ and $L$ will not deviate more than $40 \%$ from their respective theoretical values, $w$ is limited between $0.6 w_{t h}$ and $1.4 w_{t h}$, while $L$ is limited between $0.6 L_{t h}$ and $1.4 L_{t h}$. On the other hand, the maximum $F G$ is expected to be found when $d$ ranges between $0.6 \lambda$ and $0.8 \lambda$ ( $\lambda$ is the free space wavelength at $1900 \mathrm{MHz}$ ). The limits of $w, L$ and $d$ are shown in Table I. The input impedances
TABLE I

GeOMETRY PARAMETER LIMITS

\begin{tabular}{ccc}
\hline Parameter & Lower Limit $(\mathrm{mm})$ & Upper Limit $(\mathrm{mm})$ \\
\hline$w$ & 37.4 & 87.4 \\
$L$ & 30.9 & 72.1 \\
$d$ & 94.7 & 126.3 \\
$w_{1}, \ldots, w_{9}$ & 1.0 & 25.0 \\
$L_{f}, L_{9}$ & 1.0 & 39.5 \\
\hline
\end{tabular}

of the elements of the optimum MAA and the optimum value of $d$ are recorded to be used in the optimization of the MFN performed in the second (main) phase of our study.

\section{B. Microstrip Feeding Network Geometry}

The proposed MFN geometry is composed of 17 discrete microstrip line segments to feed eight array elements (see Fig. 1). Since the study aims at producing a broadside radiation pattern, this geometry is considered to be symmetric and is thus defined by the following 12 parameters:

1) nine different widths, $w_{1}, \ldots, w_{9}$,

2) the common length $L_{f}$ of the segments 1-4 along y-axis used to align the array elements along z-axis,

3 ) the common length $d$ of the segments 5-7 and the length $d / 2$ of the 8th segment along $\mathrm{z}$-axis, which ensure equal spacing between the array elements, and

4) the length $L_{9}$ of the 9th segment used for matching to an external $50 \Omega$ source.

From the above parameters, $d$ cannot be used as optimization parameter because it was recorded in phase 1 and is kept constant during phase 2 . Thus, to optimize the MFN according to the four requirements described in section I, 11 parameters $(Q=11)$ have to be optimized: $w_{1}, \ldots, w_{9}, L_{f}$ and $L_{9}$.

To help PSOvm converge faster, these parameters have to be restricted between a lower and an upper limit. The widths $w_{1}, \ldots, w_{9}$ are limited between $1 \mathrm{~mm}$ and $25 \mathrm{~mm}$ to have feasible fabrication in practice. The lower limit for $L_{f}$ and $L_{9}$ is set equal to $1 \mathrm{~mm}$, which is considered as the shortest feasible length in practice, while the upper limit is set equal to $\lambda / 4$ considering that a complete variation of voltage, current or impedance is observed along $\lambda / 4$. All these limits are summarized in Table I.

\section{PSO With Velocity Mutation}

Several PSO variants proposed so far have significantly improved the original algorithm. The idea of this particular variant (PSOvm) [17], [18] is to apply perturbation to the velocities of the particles that did not improve their fitness value in the previous iteration. Thus, it is expected that, by doing so, the swarm is protected from being trapped around a local optimum position and premature convergence is avoided. Practically, this leads to greater exploration by the swarm. PSOvm is based on the constriction coefficient PSO (CCPSO) and uses the gbest model. According to this model, one of the components used to update the velocity of a particle is influenced by the best position found by the whole swarm. The other terms include the best position found so far by the particle and its velocity at the previous iteration. By assuming 
TABLE II

MFN OPTIMIZATION RESULTS

\begin{tabular}{cccc}
\hline $\begin{array}{c}\text { Output Ports } \\
\text { (on Lumped } \\
\text { Loads) }\end{array}$ & $\begin{array}{c}\text { Relative } \\
\text { Current } \\
\text { Amplitudes } \\
\left(A_{l}\right)\end{array}$ & $\begin{array}{c}\text { Phase } \\
\text { Difference }\left(^{\circ}\right) \\
\text { with respect to } \\
\text { middle ports }\end{array}$ & $\begin{array}{c}\text { Ideal Dolph- } \\
\text { Chebyshev } \\
\text { Amplitudes } \\
\left(C_{l}\right)\end{array}$ \\
\hline $\begin{array}{c}1 \& 8 \\
2 \& 7\end{array}$ & 0.520 & 1.9 & 0.580 \\
3 \& 6 & 0.688 & -0.8 & 0.660 \\
$4 \& 5$ & 0.882 & 1.2 & 0.875 \\
& 1 & 0 & 1 \\
\hline
\end{tabular}

TABLE III

COMPlETE STRUCTURE RESUlTS

\begin{tabular}{cccc}
\hline $\begin{array}{c}\text { Output Ports } \\
\text { (Array Element } \\
\text { Inputs) }\end{array}$ & $\begin{array}{c}\text { Relative } \\
\text { Current } \\
\text { Amplitudes } \\
\left(A_{l}\right)\end{array}$ & $\begin{array}{c}\text { Phase } \\
\text { Difference }\left(^{\circ}\right) \\
\text { with respect to } \\
\text { middle ports }\end{array}$ & $\begin{array}{c}\text { Ideal Dolph- } \\
\text { Chebyshev } \\
\text { Amplitudes } \\
\left(C_{l}\right)\end{array}$ \\
\hline $\begin{array}{c}1 \text { \& } 8 \\
2 \& 7\end{array}$ & 0.532 & 0.6 & 0.580 \\
$3 \& 6$ & 0.660 & -0.1 & 0.660 \\
$4 \& 5$ & 0.880 & 2.0 & 0.875 \\
& 1 & 0 & 1 \\
\hline \multicolumn{5}{c}{$S W R=1.15$} & $P E=0.845(84.5 \%)$ \\
\end{tabular}

a swarm size of $N$ particles in a $Q$-dimensional space $(Q$ is the number of parameters to be optimized), the velocity and position of the $n$th particle $(n=1, \ldots, N)$, that improves its fitness at the $i$ th iteration, are updated using the CCPSO equation set:

$$
\begin{gathered}
v_{n q}(i+1)=k\left\{v_{n q}(i)+\phi_{1} R\left[p_{n q}(i)-x_{n q}(i)\right]\right. \\
\left.+\phi_{2} R\left[g_{q}(i)-x_{n q}(i)\right]\right\} \\
x_{n q}(i+1)=x_{n q}(i)+v_{n q}(i+1),
\end{gathered}
$$

where $v_{n q}$ and $x_{n q}$ are, respectively, the $q$ th velocity component and the $q$ th position coordinate $(q=1, \ldots, Q)$ of the $n$th particle, $p_{n q}$ and $g_{q}$ are the $q$ th coordinates of the best positions found at the end of the $i$ th iteration by the $n$th particle and the whole swarm, respectively, $R$ represents uniformly distributed random numbers within $(0,1), \phi_{1}$ and $\phi_{2}$ are, respectively, the social and the cognitive coefficient, and are both equal to 2.05 , and finally $k=0.73$ is the constriction coefficient, as explained in section III of [17]. However, in PSOvm, when a particle fails to improve its fitness at the $i$ th iteration, its velocity is updated as follows:

$$
\begin{aligned}
v_{n q}(i+1) & =k\left\{(0.6+0.1 m)(2 R-1) v_{n q}(i)\right. \\
& +\phi_{1} R\left[p_{n q}(i)-x_{n q}(i)\right] \\
& \left.+\phi_{2} R\left[g_{d}(i)-x_{n q}(i)\right]\right\}, \quad m=1, \ldots, 6,
\end{aligned}
$$

where $m$ is the number of consecutive iterations with no fitness improvement for the $n$th particle. If the $n$th particle does not improve its fitness six times in a row, then its velocity is updated with (5). This also happens when the particle manages to improve its fitness. The term $(0.6+0.1 m)(2 R-1)$ as well as the maximum value of $m$ were found (i.e., yielded the best results) after benchmarking PSOvm with several well known multi-dimensional mathematical fitness functions [17].

\section{Optimization Results}

PSOvm is used in conjunction with CST in the first two phases, to optimize MAA and the MFN, using a swarm size of 20 particles $(N=20)$. The above swarm size was decided after several MAA and MFN optimization trials, which revealed that satisfactory results may be derived when using a swarm with size $N \geq 20$. Optimization algorithms usually minimize a fitness function in order to find the optimal parameter values that correspond to a near-global fitness minimum, which in turn satisfies the requirements set either in phase 1 or in phase 2. Consequently, we define two fitness functions that incorporate the requirements set, respectively, for the optimization of MAA and the MFN:

$$
\begin{aligned}
& \text { Fit }_{1}=-F G \\
& \text { Fit }_{2}=k_{1} \max (S W R, 1.2)+k_{2} \min (P E, 0.8) \\
& +k_{3}\left[\operatorname{abs}\left(A_{1}-C_{1}\right)+\operatorname{abs}\left(A_{2}-C_{2}\right)+a b s\left(A_{3}-C_{3}\right)\right] \\
& +k_{4} \max \left[\operatorname{abs}\left(p_{1}-p_{2}\right), \operatorname{abs}\left(p_{1}-p_{3}\right), \operatorname{abs}\left(p_{1}-p_{4}\right),\right. \\
& \left.\quad \operatorname{abs}\left(p_{2}-p_{3}\right), \operatorname{abs}\left(p_{2}-p_{4}\right), \operatorname{abs}\left(p_{3}-p_{4}\right), 3\right],
\end{aligned}
$$

where $F G$ is given in $\mathrm{dBi}, A_{l}(l=1, \ldots, 3)$ is the relative current amplitude at the $l$ th output port of the MFN with respect to the current amplitude at the 4th port (thus $A_{4}=1$ ), $C_{1}, \ldots, C_{3}$ are the ideal relative amplitudes $\left(C_{4}=1\right)$ defined by D-Ch distribution for $S L L=-20 \mathrm{~dB}, p_{1}, \ldots, p_{4}$ are the phases of the output currents (in degrees), and finally $k_{1}, \ldots, k_{4}$ are positive weights used to equalize the magnitudes of all terms of $\mathrm{Fit}_{2}$, so that they all have the same priority during the optimization process. Obviously, due to the symmetry of the structure, the four remaining output ports on the right side of Fig. 1 have the same current amplitudes and phases with their symmetric ones on the left side of Fig. 1, and for that reason these amplitudes and phases are not used in Fit $_{2}$. To help the optimization algorithm achieve current amplitudes as close as possible to the ideal D-Ch distribution, as it is the main factor that ensures low SLL, we use $\max$ functions for the $S W R$ (1st fitness term) and the phase differences (4th fitness term) as well as a min function for the PE (2nd fitness term), so that values of $S W R$ below 1.2, values of $P E$ above 0.8 and phase differences below $3^{\circ}$ will not affect Fit $_{2}$ and therefore the algorithm will focus only on achieving D-Ch current amplitudes.

Phase 1 results in a MAA with $F G=15.4 \mathrm{dBi}$ and provides the data needed for the next phase. Since full wave analysis is performed on MAA during the optimization procedure of phase 1 and the mutual coupling between the elements of the optimum MAA is taken into account, the input impedances of these elements are derived with complex values different from each other. The results of phase 2 (MFN optimization) are displayed in Table II and it can be seen that all the requirements are satisfied. Finally in phase 3 (evaluation phase), the lumped loads are removed from the MFN, MAA is attached to the MFN, full wave analysis is applied to the complete structure (with CST), and again the currents at the array element inputs are recorded. The results are shown in Table III. It seems that the requirements set for the MFN are met, while the $F G$ and $S L L$ of the complete structure are in agreement with the 
TABLE IV

Complete Structure Geometry

\begin{tabular}{cc|cc}
\hline Parameter & Value $(\mathrm{mm})$ & Parameter & Value $(\mathrm{mm})$ \\
\hline$w_{1}$ & 2.1 & $w_{8}$ & 11.3 \\
$w_{2}$ & 5.3 & $w_{9}$ & 18.3 \\
$w_{3}$ & 10.0 & $L_{9}$ & 25.0 \\
$w_{4}$ & 10.8 & $L_{f}$ & 20.1 \\
$w_{5}$ & 6.9 & $L$ & 45.3 \\
$w_{6}$ & 21.6 & $w$ & 84.9 \\
$w_{7}$ & 15.0 & $d$ & 109.0 \\
\hline
\end{tabular}

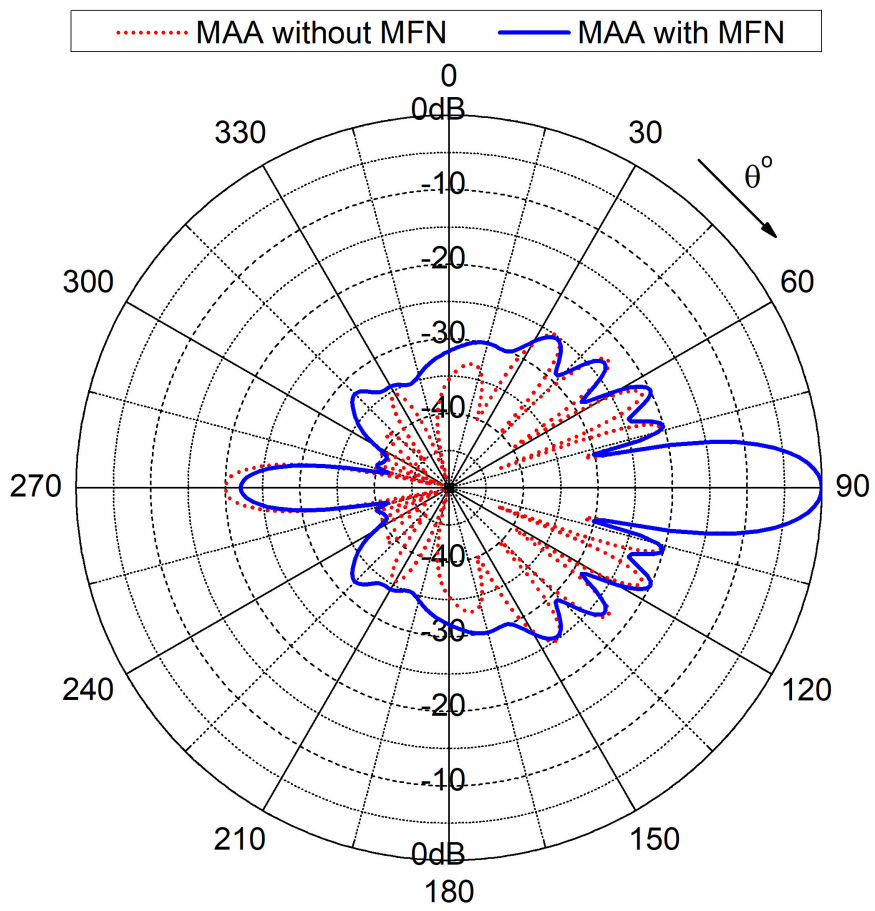

Fig. 2. Radiation patterns with and without the MFN.

respective values of MAA derived in phase 1 . There is a small deviation from the relative amplitudes and phase differences obtained in phase 2 , but this is expected, because between the MFN and MAA there is a small coupling, which does not exist between the MFN and the lumped loads. To further support our claim, that the MFN can be designed separately from MAA, we compare the radiation patterns obtained with and without the presence of the MFN. It can be seen in Fig. 2 that the radiation patterns are in good agreement. The dimensions of MAA (derived in phase 1) and the MFN (derived in phase 2) are summarized in Table IV.

Finally we evaluate the characteristics of the complete optimized structure (i.e., the optimized MAA together with the optimized MFN) in terms of fabrication errors. For this purpose, we run $1000 \mathrm{CST}$ models of the complete structure, where we apply random concurrent deviations $\pm 1 \%$ at most in the values of all the geometry parameters of this structure. In this way, we calculate the standard deviation of the structure characteristics with respect to their optimized values. The results are shown in Table $\mathrm{V}$ and reveal that actually there are no significant differences from the optimized structure characteristics if fabrication errors occur.
TABLE V

Standard DeViation of Final Structure Characteristics With FABRICATION ERROR $\pm 1 \%$

\begin{tabular}{cc|cc}
\hline Parameter & Std & Parameter & Std \\
\hline$A_{1}$ & 0.012 & $S W R$ & 0.264 \\
$A_{2}$ & 0.007 & $P E$ & 0.041 \\
$A_{3}$ & 0.017 & $F G$ & $0.1 \mathrm{~dB}$ \\
$p_{1}-p_{4}$ & $1.0^{\circ}$ & $S L L$ & $0.5 \mathrm{~dB}$ \\
$p_{2}-p_{4}$ & $0.8^{\circ}$ & Bandwidth & $<1 \mathrm{MHz}$ \\
$p_{3}-p_{4}$ & $0.6^{\circ}$ & & \\
\hline
\end{tabular}

\section{CONCLUSION}

An MFN with equiphase D-Ch current distribution, low $S W R$ and high $P E$ has been designed and all the requirements were satisfied. Moreover, it has been proven that for any MAA, an MFN that creates a D-Ch current distribution at the MAA ports can be designed separately from MAA, by only knowing the input impedances of the array elements and the spacing between them. Finally, the whole design process can be generalized for different MFN geometries and more complex MAA structures.

\section{REFERENCES}

[1] D. H. Lee, Y. J. Lee, J. Yeo, R. Mittra, and W. S. Park, "Design of metamaterial superstrates and substrates for directivity and port isolation enhancement of a dual-frequency dual-polarization microstrip antenna," Microwave and Optical Technology Letters, vol. 48, no. 9, pp. 18731876, 2006.

[2] A. U. Zaman, L. Manholm, and A. Derneryd, "Dual beam phased array antenna with wide scan angle for repeater applications," in Proc. 2008 International Conference on Electrical and Computer Engineering, Dec. 2008, pp. 755-759.

[3] B. Elnour and D. Erricolo, "A novel colocated cross-polarized twoloop PCB antenna in the ISM 2.4-GHz band," IEEE Antennas Wireless Propag. Lett., vol. 9, pp. 1237-1240, 2010.

[4] J. Liu, K. P. Esselle, and S. Zhong, "A miniaturised printed monopole antenna with an extremely wide bandwidth," in Proc. 40th European Microwave Conference, Sep. 2010, pp. 850-853.

[5] F. Elmegri, C. H. See, R. A. Abd-Alhameed, C. Zebiri, and P. S. Excell, "Dielectric resonator antenna design for UWB applications," in Proc. 2013 Loughborough Antennas Propagation Conference, Nov. 2013, pp. $539-542$.

[6] S. M. Abbas, Y. Ranga, and K. P. Esselle, "Stub-loaded printed antenna with a ground plane and electromagnetically coupled feed for $2.45 \mathrm{GHz}$ body area networks," in Proc. 2013 IEEE MTT-S International Microwave Workshop Series on RF and Wireless Technologies for Biomedical and Healthcare Applications, Dec. 2013, pp. 1-3.

[7] M. A. Elmansouri, J. B. Bargeron, and D. S. Filipovic, "Simply-fed four-arm spiral-helix antenna," IEEE Trans. Antennas Propag., vol. 62, no. 9, pp. 4864-4868, Sep. 2014

[8] R. Sammeta and D. S. Filipovic, "Reduced size planar dual-polarized log-periodic antenna for bidirectional high power transmit and receive applications," IEEE Trans. Antennas Propag., vol. 62, no. 11, pp. 54535461, Nov. 2014

[9] M. A. Elmansouri, E. A. Etellisi, and D. S. Filipovic, "Ultra-wideband circularly-polarized simultaneous transmit and receive (STAR) antenna system," in Proc. 2015 IEEE International Symposium on Antennas and Propagation USNC/URSI National Radio Science Meeting, July 2015, pp. $508-509$.

[10] C. Gu, S. Gao, H. Liu, Q. Luo, T. Loh, M. Sobhy, J. Li, G. Wei, J. Xu, F. Qin, B. Sanz-Izquierdo, and R. A. Abd-Alhameed, "Compact smart antenna with electronic beam-switching and reconfigurable polarizations," IEEE Trans. Antennas Propag., vol. 63, no. 12, pp. 5325-5333, Dec. 2015.

[11] M. A. Elmansouri, A. J. Kee, and D. S. Filipovic, "Wideband antenna array for simultaneous transmit and receive (STAR) applications," IEEE Antennas Wireless Propag. Lett., vol. 16, pp. 1277-1280, 2017.

[12] I. T. Elfergani, J. Rodriguez, F. Abdulssalam, C. H. See, and R. A. Abd-Alhameed, "Miniaturized balanced antenna with integrated balun for practical LTE applications," Radioengineering, vol. 26, no. 2, 2017. 
[13] J. Ha and D. S. Filipovic, "Electrothermal design of bidirectional wideboom log-periodic antennas," IEEE Trans. Antennas Propag., vol. 65 , no. 4, pp. 1661-1669, April 2017.

[14] Q. Wu, J. Yin, C. Yu, H. Wang, W. Hong, and J. Hirokawa, "Millimeterwave wideband circularly polarized antenna arrays using travellingwave elements," in Proc. 2018 International Workshop on Antenna Technology, March 2018, pp. 1-4.

[15] R. C. Hansen, Phased Array Antennas, 2nd ed. John Wiley \& Sons, 2009, pp. 53-60.

[16] CST Microwave Studio. [Online]. Available: http://www.cst.com

[17] Z. D. Zaharis, I. P. Gravas, T. V. Yioultsis, P. I. Lazaridis, I. A. Glover, C. Skeberis, and T. D. Xenos, "Exponential log-periodic antenna design using improved particle swarm optimization with velocity mutation," IEEE Trans. Magn., vol. 53, no. 6, pp. 1-4, June 2017.

[18] Z. D. Zaharis, I. P. Gravas, P. I. Lazaridis, I. A. Glover, C. S. Antonopoulos, and T. D. Xenos, "Optimal LTE-protected LPDA design for DVB-T reception using particle swarm optimization with velocity mutation," IEEE Trans. Antennas Propag., vol. 66, no. 8, pp. 39263935, Aug. 2018.

[19] T.-S. Horng and N. G. Alexopoulos, "Corporate feed design for microstrip arrays," IEEE Trans. Antennas Propag., vol. 41, no. 12, pp. 1615-1624, Dec. 1993.

[20] Z. Zaharis, E. Vafiadis, and J. N. Sahalos, "On the design of a dual-band base station wire antenna," IEEE Antennas Propag. Mag., vol. 42, no. 6, pp. 144-151, Dec. 2000.

[21] Z. Ahmed, M. B. Ihsan, N. Chaudhry, and J. Kiani, "Design of a low side lobe aperture coupled microstrip antenna array," in Proc. 8th International Multitopic Conference, Dec 2004, pp. 227-231.
[22] M. Liu, Z.-R. Feng, K. Zhang, and Q. Wu, "A millimeter-wave conical conformal low sidelobe microstrip antenna array," in Proc. International Conference on Microwave and Millimeter Wave Technology, vol. 4, April 2008, pp. 1810-1813.

[23] Z.-X. Zhang, M.-G. Fu, and C. Chen, "A $24 \mathrm{GHz}$ microstrip array antenna," in Proc. 2011 International Conference on Computer Science and Network Technology, vol. 1, Dec. 2011, pp. 214-217.

[24] J. Puskely, Z. Raida, and L. Slama, "Planar antenna arrays for long range links," in Proc. 6th European Conference on Antennas and Propagation, March 2012, pp. 2928-2932.

[25] B. Kasi and C. K. Chakrabarty, "Ultra-wideband antenna array design for target detection," Prog. Electromagn. Res., vol. 25, pp. 67-79, 2012.

[26] G. F. Hamberger, S. Trummer, U. Siart, and T. F. Eibert, "A planar dualpolarized microstrip antenna array in series-parallel feed configuration," in Proc. 2015 Loughborough Antennas Propagation Conference, Nov. 2015 , pp. $1-4$.

[27] H. Mardani, J. Nourinia, C. Ghobadi, M. Majidzadeh, and B. Mohammadi, "A compact low-side lobes three-layer array antenna for X-band applications," AEU-International Journal of Electronics and Communications, vol. 99, pp. 1-7, 2019.

[28] C. A. Balanis, Antenna Theory: Analysis and Design, 3rd ed. New York, NY, USA: Wiley-Interscience, 2005, pp. 816-820.

[29] Rogers Corporation. [Online]. Available: https://www.rogerscorp.com/ 